\title{
RNA-binding protein 39: a promising therapeutic target for
}

\section{cancer}

\author{
Caipeng $\mathrm{Xu}^{1,2,3,4}$, Xiaohua Chen ${ }^{1,2,3,4}$, Xuetian Zhang ${ }^{1,2,3,4}$, Dapeng Zhao ${ }^{1,2,3,4}$, Zhihui Dou ${ }^{1,2,3,4}$, Xiaodong Xie ${ }^{5}$, Hongyan Li ${ }^{1,2,3,4}$, \\ Hongying Yang ${ }^{6}$, Qiang $\mathrm{Li}^{1,2,3,4,7,8}{ }^{凶}$, Hong Zhang ${ }^{1,2,3,4,7,8}{ }^{凶}$ and Cuixia $\mathrm{Di}^{1,2,3,4,7,8}{ }^{\bowtie}$
}

(c) The Author(s) 2021

\begin{abstract}
RNA-binding motif protein 39 (RBM39), as a key factor in tumor-targeted mRNA and protein expression, not only plays a vital role in tumorigenesis, but also has broad development prospects in clinical treatment and drug research. Moreover, since RBM39 was identified as a target of sulfonamides, it has played a key role in the emerging field of molecule drug development. Hence, it is of great significance to study the interaction between RBM39 and tumors and the clinical application of drug-targeted therapy. In this paper, we describe the possible multi-level regulation of RBM39, including gene transcription, protein translation, and alternative splicing. Importantly, the molecular function of RBM39 as an important splicing factor in most common tumors is systematically outlined. Furthermore, we briefly introduce RBM39's tumor-targeted drug research and its clinical application, hoping to give reference significance for the molecular mechanism of RBM39 in tumors, and provide reliable ideas for in-depth research for future therapeutic strategies.
\end{abstract}

Cell Death Discovery (2021)7:214; https://doi.org/10.1038/s41420-021-00598-7

\section{FACTS}

- RBM39 is characterized by upregulated expression in many cancers, but the precise molecular function in specific cancers may not be thorough enough.

- RBM39 is indirectly involved in tumor growth and progression by regulating transcription of many tumor-related genes, protein translation, and alternative splicing.

- RBM39 can be exploited as markers for more accurate diagnosis, as well as improved prognosis and monitoring of treatment response in patients with cancer.

- RBM39 is identified as a target of sulfonamides, and it has played a key role in the emerging field of small molecule drug development.

\section{OPEN QUESTIONS}

- What is the exact molecular function of RBM39 in normal and cancer tissues?

- What is the preferential effect of RBM39 degradation in cancer cells?

- Would SPLAMs be used in more research tests and clinical patient treatments to improve the potential clinical efficacy of SPLAMs?
- Would SPLAM derivatives recruit new substrates other than RBM39 to join CUL4-DCAF15 to better exert the clinical value of the drug?

\section{INTRODUCTION}

Cancer has always been regarded as a highly complex disease, which seriously threatens human health and life. People have been committed to the treatment and clinical research of cancer for a long time, so it is necessary to deeply understand the molecular mechanism of cancer progression and research on targeted drugs. Here, RNA-binding motif protein 39 (RBM39), also known as splicing factor HCC1, CAPERa, FSAP59, RNPC2, and CAPER alpha, is an essential serine/arginine-rich (SR) RNA-binding protein, as well as a pre-mRNA splicing factor and transcription coactivator. RBM39 was initially identified as the autoantigen from a patient with liver cirrhosis who later developed hepatocellular carcinoma [1]. With in-depth research on the characteristics and multiple functions of RBM39, it is revealed that RBM39 is mainly involved in biological processes, such as transcriptional regulation, alternative splicing, and protein translation [2]. An increasing body of data suggests that RBM39 has been closely linked to malignant progression of various cancers, and its expression may be related to RBM39 mutual proteins in specific environments. Notably, RBM39 is upregulated in most cancers, and the inhibition of its

\footnotetext{
${ }^{1}$ Bio-Medical Research Canter, Institute of Modern Physics, Chinese Academy of Sciences, Lanzhou 730000, China. ${ }^{2}$ Key Laboratory of Heavy lon Radiation Biology and Medicine of Chinese Academy of Sciences, Lanzhou 730000, China. ${ }^{3}$ College of Life Sciences, University of Chinese Academy of Sciences, Beijing 101408 , China. ${ }^{4}$ School of Nuclear Science

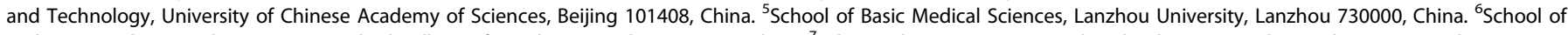

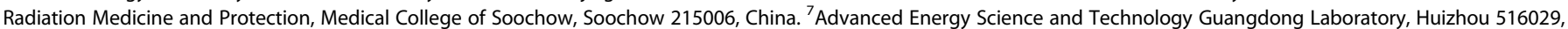
China. ${ }^{8}$ These authors contributed equally: Qiang Li, Hong Zhang, Cuixia Di. ${ }^{凶}$ email: liq@impcas.ac.cn; zhang.h@impcas.ac.cn; dicx@impcas.ac.cn Edited by Dr Maria Victoria Niklison Chirou
}

Received: 30 November 2020 Revised: 4 March 2021 Accepted: 29 May 2021

Published online: 13 August 2021 
Table 1. Research application of RBM39 in tumor.

\begin{tabular}{ll}
\hline Cancer & Expression \\
\hline Ewing Sarcoma & Up \\
\hline Breast cancer & Up \\
\hline TNBC & Up \\
\hline Invasive ductal carcinoma & Up \\
\hline Colorectal adenoma & Up \\
\hline Hepatocellular carcinoma & Up \\
\hline Prostate cancer & Up \\
\hline Myeloma malignancy & Down \\
\hline AML & Up \\
\hline Lung cancer & Up \\
\hline
\end{tabular}

function is lethal to several cancers including lung cancer [3], breast cancer [4], and colorectal cancer [5]. For instance, reducing the expression of RBM39 can inhibit the proliferation of estrogen receptor (ER)-positive human breast cancer cells [6, 7], which further suggests that RBM39 is an important signaling molecule in the pathogenesis of breast cancer, which may bring new methods for treatment of invasive cancer [4]. Interestingly, RBM39 is a potential tumor suppressor in few human malignancies. RBM39 could effectively recede the carcinogenic activity of NF-kb-Rel protein in lymphocytes [8], and its overexpression inhibited tumor angiogenesis and growth [9]. Therefore, small-molecule drugs targeting RBM39 may be an extremely valuable method in the current treatment of various malignant tumors.

In recent years, although there have been relevant reports on the research of RBM39 in tumorigenesis and development, the precise function in specific cancers may not be thorough enough. In addition, due to the limited efficacy of sulfonamides, its research progress in clinical trials is relatively slow, so it is very important to try to reveal its latest research progress in tumors. In this review, we mainly summarized the possible regulation mechanism of RBM39 involved in tumor, and briefly discussed the molecular function of RBM39 in tumors. Furthermore, we outlined the emerging tumor-targeted drug research and its clinical application. Thus, it is highlighting the significance of RBM39 as targets for cancer therapy.

\section{CHARACTERISTICS OF RBM39}

RBM39 has significant sequence similarity with splicing factors U2AF65, and RBM23 (Table 1), and contains both R-X-F elements and U2AF homology motif (UHM) negatively charged a-helix A $[1,2,10]$. It is similar to RBM23 and U2AF65 shares the N-terminal RS domain together with RBM23 and PUF60, and shares RRM1/RRM2 and C-terminal UHM [11]. In particularly, U2AF65 contains the U2AF ligand substrate, others do not [12] (Table 1A). HCC1/RBM39 contains two alternately spliced subtypes, called HCC1.3 and HCC1.4. HCC1.4, a full-length cDNA clone encoding 530 amino acids, can interact with the C-terminus of the SR-related protein SRrp53, and SRrp53 has been shown to activate the $3^{\prime}$ weak splice site [13]. HCC1.3 is another representative clone of RBM39, and its 18 nucleotide (six amino acids) deletion is located in the third RNA-recognition motif (RRM) domain, which can affect the splicing reaction $[1,14,15]$. It has been revealed that HCC1.4 and HCC1.3 as TAAs have different autoantibody immune responses [3], but they are not distinguishable in terms of binding and transcriptional coactivation properties. Additionally, RBM39 mRNA exhibitings obvious tissue specificity in normal tissues has been discovered. For example, it is highly transcribed in CD56 + natural killer cells, CD4 + and CD8 + T cells, CD19 + B lymphocytes, CD34 + cells and CD33 + myeloid cells, and

\section{Related Function}

Refs

Regulates proliferation and protein synthesis pathways

Mediates VEGF alternative splicing

Regulates DNA repair, cell apoptosis and cell cycle

Transfers from the cytoplasm to the nucleus

Mediates cell viability

Affects cell survival and viability

Related to the appearance of microvessels

Cancer cell migration and invasion

Regulates mTOR signaling, promotes cell proliferation

Causes changes in the splicing of HOXA9 target genes

Promotes proliferation and migration is expressed in large amounts in immune system-related cells, lymphnode cells, uterine cells, and thyroid cells [16]. In brief, the characteristics and tissue specificity of RBM39 determine that it may be involved in a variety of biological processes.

\section{REGULATION MECHANISM OF RBM39} RBM39 coordinates transcriptional regulation

It has been previously demonstrated that RBM39 is involved in transcriptional regulation of related proteins and various genes (Fig. 1B, Fig. 2). RBM39 can bind to the transcription factor activating protein-1 (AP-1)/c-Jun and the nuclear steroid receptors ER- $\alpha$ and ER- $\beta$ in vivo and effectively promote its transcription [2, 14, 17]. In addition, it interacts with ASC-2, a coactivator that may act to recruit molecules containing RRM domains (Fig. 1B). Dowhan et al. pointed out that RBM39 could interact with progesterone receptor (PR) in vitro and stimulate the transcriptional activity of hormonemediated PR [2]. Interestingly, RBM39 had high selectivity for c-Jun, $E R a / \beta$, and $P R$, and did not have an effect on other transcription factors, such as p53, serum-response factor, c-fos, NF-kB component $\mathrm{p} 50$, glucocorticoid hormone receptor, farnesoid $X$ receptor, thyroid hormone receptor- $\alpha / \beta$, and liver $X$ receptor- $\alpha / \beta$, among others $[2,8,14]$. It is reported that RBM39 had higher transcript levels in brain cancer and ovarian cancer [18]. Additionally, the study revealed that a total of 304 differentially expressed genes in RBM39-deficient cells were significantly regulated by RBM39, of which 123 were downregulated and 181 were upregulated, indicating that RBM39 could affect the regulation of transcription levels [19]. Furthermore, Mai et al. investigated that as a substrate of tyrosine kinase, RBM39 had a structural interaction with c-Abl [20]. Importantly, c-Abl could phosphorylate RBM39 and promote the transcriptional coactivation activity of RBM39 for ERa and PR $\beta$, making it highly expressed in the placenta and liver tissues of the steroid hormone-dependent signaling pathway [20]. It is considered that RBM39 induced nuclear genes by coactivating ERR-a-Gabpa, which could directly lead to transcriptional regulation of regulators, including C-MYC and Gabpa [21]. Kumar et al. revealed that RBM39 could interact with TBX3 to inhibit the transcription of CDKN2A$\mathrm{p}^{1} 6^{\mathrm{INK}}$ and $\mathrm{Rb}$ pathways. Therefore, RBM39/TBX3 and UCA1 constituted an important mechanism to regulate the transcription of CDKN2A-p16 ${ }^{\text {INK }}$ [22]. In general, RBM39 enhances the activity of transcription factors and participates in the formation of the initiation complex through the interaction between transcription factors that bind to promoters [2].

\section{RBM39 regulates protein translation}

It is demonstrated that RBM39 binds to mRNAs related to coding and translation proteins and participates in the expression of translation-related proteins [23], but there are currently not many 
A

\begin{tabular}{|c|c|c|c|}
\hline Gene name & Protein name & Protein domain & Protein length \\
\hline RBM39 & $\operatorname{CAPERa}(20 \mathrm{q} 11.3)$ & RS - RRM1 - RRM2 - UHM & $530 a a$ \\
\hline RBM23 & CAPERß(14q 11.2) & RS RRM1 & $439 a a$ \\
\hline U2AF2 & U2AF65(19q 13.43) & RS - ULM - RRM1 - RRM2 - UHM & $475 a a$ \\
\hline PUF60 & PUF60(8q 24.2) & RRM1 - RRM2 — UHM & $559 a a$ \\
\hline
\end{tabular}

B

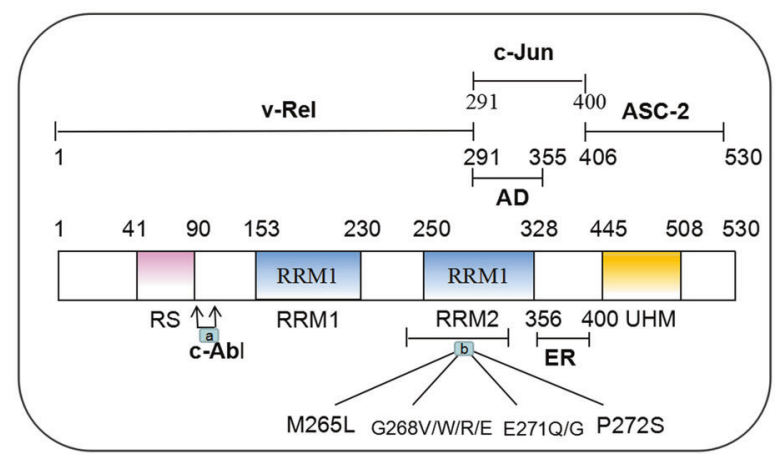

C

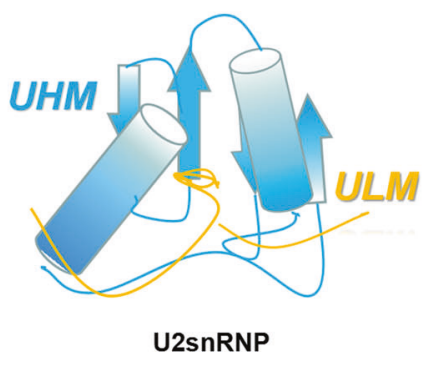

Fig. 1 The domain of RBM39 and its related proteins. A The domain composition of U2AF65 and its paralogous proteins. B Different functional domains of RBM39 (a. The interaction between c-Abl and RBM39, the phosphorylation site of RBM39; b. The toxic effect of indisulam, the mutation domain of indisulam gathers in the RRM2 domain of RBM39). C Schematic of the UHM-ULM-interaction structure.

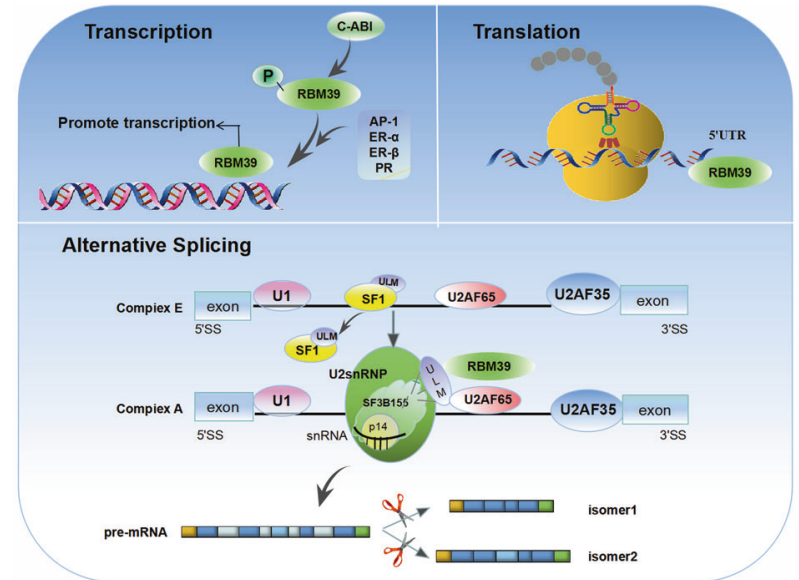

Fig. 2 Schematic diagram of the function of RBM39 in cancer cells. RBM39 participates in the regulation of gene expression from multiple levels, including transcription, alternative splicing, and translation.

such reports. Critically, the binding of RBM39 at the 5'UTR site could affect protein translation, whereas particularly under conditions of this special combination of $5^{\prime} / 3^{\prime}$ splice sites, it might be more conducive to selective splicing regulation [19]. Mai et al. found that the genes located at the binding peak of RBM39 were enriched in protein translation, and through in vitro translation experiments proved that the translation of luciferase mRNA was significantly inhibited after the RBM39 gene was knocked out [19]. Meanwhile, it is well established that the downregulation of RBM39 activity reduced the expression of cell cycle advancement regulators and inhibited protein-synthesis pathways, thereby affecting the proteintranslation process [6]. In addition, current evidence suggests that RBM39 undergoes post-translational modifications, including ubiquitination and phosphorylation. Depending on this, RBM39 plays an extremely critical role in regulating protein translation in multiple cell processes.

\section{RBM39 regulates alternative splicing}

Alternative splicing is a highly regulated and coordinated molecular mechanism involving multiple physiological processes [24]. RBM39 has been considered as a potential splicing factor, which plays an important role in pre-mRNA alternative splicing of human, yeast and Drosophila $[25,26]$, It could colocalize with splicing factors SRSF2, $\mathrm{SC} 35$, and $\mathrm{m} 3 \mathrm{G}$ at the nuclear spot, which was rich in pre-mRNA splicing proteins $[1,15]$. As the U2snRNP related splicing factor $3 \mathrm{~b}$ subunit 155 (SF3b155) contains U2AF ligand motif (ULM), RBM39 could interact with U2AF65 [27, 28], SF3B155 [27, 28], and RSRC1 [13], which might provide an opportunity to regulate alternative splicing. RBM39 combined with essential splicing factors in specific complexes through UHM/ULM interaction, promoted the recruitment of U2snRNP and the coordination of splicing factors, so as to form splice complexes and splice pre-mRNA species. Therefore, RBM39 might interact with splicing factors related to early recognition of $3^{\prime}$ splice sites, thereby affecting the progress of splicing reactions.

Alternative splicing of RBM39 is an extremely important mechanism, and its regulatory mechanism is similar to that of U2AF65, which can directly bind to RNA or recruit specific splicing factors to regulate selective splicing. It was further revealed that as many as $20 \%$ of alternative splicing exons were regulated by both RBM39 and U2AF65 [19]. Mai et al. studied RBM39-mediated alternative splicing in human breast cancer MCF-7 cells and found a total of 359 RBM39regulated alternative splicing events, of which cassette exons were the most common regulatory target [19]. Moreover, genes regulated by RBM39-mediated alternative splicing were involved in G2/M cell cycle transition and cell response to DNA damage [6, 19]. Regarding the regulation of alternative splicing of RBM39, it was further confirmed that in T47D breast cancer cells, downregulated expression of RBM39 could correspondingly change the alternative splicing of vascular endothelial growth factor (VEGF) and increase the ratio of VEGF121 and VEGF189 in breast cancer [14]. Huang et al. demonstrated in Ewing cells that the overexpression of RBM39 mediated the alternative splicing of VEGF and controlled the transition from VEGF189 to VEGF165, while the downregulation of RBM39 led to an increase in the ratio of VEGF165/VEGF189 [9]. Subsequently, Taisuke et al. confirmed that VEGF189 mRNA was significantly decreased and 
VEGF121mRNA was increased after treatment with these sulfonamides or RBM39 siRNA [29]. The splicing regulator polypyrimidine tract-binding protein inhibited the excision of alternatively spliced exons by preventing U2AF from binding at the $3^{\prime}$ splice site and $5^{\prime}$ splice site dependently [30]. In addition, RBM39 promoted the inclusion of false exons in the iron-sulfur cluster assembly gene by inhibiting the binding to polypyrimidine tract-binding protein, thereby affecting the specific splicing selection of RBM39 [31]. Interestingly, RBM39 is also thought to play an important role in SIN3B alternative splicing. Studies identified that BMP4-dependent transcription was regulated by targeted negative feedback of SIN3B alternative splicing, which could enhance BMP activity after knocking out RBM39 [32]. Taken together, as a vital splicing factor, RBM39 participates in a wide range of alternative splicing of pre-mRNA. Importantly, based on its biological role and clinical significance in tumor progression, it might bring new ideas for elucidating the mechanism of tumor occurrence, development, and metastasis. Nevertheless, the extent to which RBM39 is involved in alternative splicing is still unknown, so it is important to explore the regulatory mechanism of RBM39 alternative splicing.

\section{MOLECULAR FUNCTION OF RBM39 IN CANCER \\ Breast cancer}

A large number of data suggested that RBM39 regulated the transcriptional activity of ERs and PRs and was involved in the progression of human breast cancer [4, 6, 33]. RBM39 was expressed at high levels in human breast cancer tissues, but it was almost undetectable in normal breast tissues [6]. In addition, RBM39 was also overexpressed in mouse models of breast cancer and was associated with increased estrogenic sensitivity, indicating that it could play an important role in the early development of ER-positive breast cancer [6]. Mercier et al. found that RBM39 expression was positively correlated with tumor size in vitro and in vivo, and virus-mediated knockdown of RBM39 in ER-positive MCF-7 cells could significantly hinder the proliferation of human breast cancer cells and tumor growth in vivo [6]. To a certain extent, it might also inhibit AP-1/c-Jun transcriptional activity in MCF-7 cells, thereby inhibiting the phosphorylation of c-Jun [6,34]. Regarding the mechanism, knockdown of RBM39 inhibited cell proliferation and protein-synthesis pathways [6]. Another study documented that the expression of RBM39 was significantly upregulated in human triple-negative breast cancer (TNBC) specimens compared with normal breast tissue. If the expression of RBM39 was inhibited, the proliferation of human breast cancer cells could be significantly reduced[4]. Furthermore, in terms of mechanism, knockdown of RBM39 could impair the functional repair of DNA by inhibiting the activity of RAD51, c-Abl and Rb proteinsynthesis pathways, and then induce apoptosis. It can be seen that RBM39 is expected to become a potential therapeutic target and biomarker for TNBCs to predict the response to DNA damage treatment. Through the analysis and identification of human breast cancer samples, it was found that the overexpression of RBM39 experienced cytoplasmic-to-nuclear metastasis in the process of breast cancer metastasis from pre malignant to ductal carcinoma [35]. It follows that RBM39 has extremely broad development prospects in the treatment of this aggressive cancer.

\section{Acute myeloid leukemia}

Acute myeloid leukemia (AML) is an aggressive hematological malignant tumor with a high recurrence rate after conventional combined chemotherapy [36]. Regarding the treatment of AML, one aspect was dedicated to mutation-specific targeted drug research [37], and the other was to identify other nonmutant proteins that were necessary for the survival of AML cells. Among them, H3b-8800 was one of these splicing factor inhibitors, which was currently in phase I clinical trial of refractory myeloid malignancies [38]. Proteomics studies found that there was an important RBP splicing network in AML, as a key member of the RBP. RBM39 was closely dependent on cancer [39]. In particular, degradation of RBM39 led to abnormal splicing of transcriptional regulators necessary for the survival of $A M L$, thus playing an extremely critical role in maintaining RNA splicing and survival of AML. Recently, Wang et al. revealed that knocking out RBM39 could slow the progression of leukemia and improve overall survival. Notably, the anticancer effects of RBM39 gene knockout in indisulam treatment were studied in vitro and in vivo, and SPlicing inhibitor sulfonamides (SPLAMs) were found to be an effective and safe AML treatment with minimal adverse effects on hematopoietic cells [40]. Moreover, follow-up studies clarified the mechanism by which CRISPR-mediated degradation of RBM39 led to incorrect splicing of HOXA9 target genes and extensive antileukemia effects [41]. In general, RBM39 is critical for AML cell survival and disease progression.

\section{Lung cancer}

RBM39 plays an important role in the occurrence and development of lung cancer. With its tumor-specific immune function, RBM39 has a unique role in regulating the biological functions of tumor cells $[35,42]$. Kumar et al. confirmed that there were at least two RBM39 subtypes that could be used as tumorassociated antigens, which might stimulate the humoral immune response in human lung cancer patients [3]. Bangur et al. found 209 genes and nine initial characteristics of genes by identifying overexpressed genes in small-cell lung cancer, among which RBM39 was differentially expressed in nonsmall-cell lung cancer (NSCLC), which provided valuable basis for better understanding of the biological characteristics of NSCLC [42]. In order to further explore the role of RBM39 in the growth and migration of NSCLC cells, studies have shown that RBM39 was mainly located in the nucleus of lung cancer cells. After a series of analyses, it was found that the expression frequency of RBM39 in NSCLC tissues was significantly higher than that in adjacent tissues and normal tissues. Moreover, the overexpression of RBM39 could promote the proliferation and migration of NSCLC cells, and the expression of RBM39 had no obvious correlation with tumor stage, gender, and age, which preliminarily revealed that RBM39 was expected to become a biomarker and potential therapeutic target for lung cancer [3].

\section{Myeloma malignancy}

As a new oncogene in myeloma, RBM39 is essential for the proliferation of multiple myeloma cells and tumorigenesis. Based on its biological role and clinical significance in tumor progression, RBM39 might become a key prognostic indicator for multiple myeloma patients [43]. Tong et al. implicated that RBM39 was highly expressed in myeloma cells, and its expression level was related to the poor prognosis of the tumor. Under hypoxia, knockout of RBM39 gene inhibited the mTOR signaling pathway, which could be reversed by RBM39 overexpression. In addition, further studies have shown that RBM39 inhibited the ubiquitination and degradation of RBM39 protein through the interaction with DARS-AS1, indicating that RBM39 might mediate the biological function of DARS-AS1 on multiple myeloma under hypoxic conditions [44]. Therefore, targeting the RBM39 axis might be a potential therapeutic target for multiple myeloma.

\section{Other cancers}

According to previous studies, RBM39 is considered to be a novel antigen identified from patients with hepatocellular carcinoma, showing high levels of expression in hepatocellular carcinoma $[1,14]$. It pointed out that anti-RBM39 autoantibody was the highest in liver cancer, and its immunostaining intensity in liver cancer cells was significantly reduced, and it was positively correlated with its decreased expression level, which further indicated that the decrease in RBM39 expression level might be related to the appearance of microvessels [45]. Additionally, RBM39 has also been reported in colorectal cancer and esophageal cancer. 
Anke $\mathrm{H}$ et al. found that RBM39 located on the 20Q-amplified fragment was upregulated in human colorectal adenoma and colorectal cancer, and affected cell survival and anchoringindependent growth by participating in some cancer-related biological processes [5]. Another study showed that the upregulated expression of RBM39 significantly increased the survival rate of colorectal cancer cells, suggesting that RBM39 has an extremely important potential function in apoptosis [46].

\section{OPTIONS MODULATING RBM39 EXPRESSION IN CANCER Anticancer sulfonamides}

Recent studies revealed that SPLAMs, including indisulam, E7820, chloroquinoxaline sulfonamide (CQS), and tasisulam (Fig. $3 \mathrm{~A}$ ), are degradation products of RBM39, and DCAF15 was the main target of ubiquitination of SPLAMs $[47,48]$. Therefore, SPLAMs could recruit splicing factor RBM39 to E3 ligase substrate receptor DCAF15, and promoted the proteasomal degradation of RBM39 through CRL4DCAF15-mediated ubiquitination, leading to splicing abnormalities of pre-mRNA dominated by intron retention and exon skipping [49] (Fig. 4). The main ubiquitination sites of RBM39 were located in the $\mathrm{N}$-terminal region of 120 amino acids [29]. Moreover, SPLAMs used a mechanism similar to immunomodulatory drug (IMiDs) to target pre-mRNA splicing. IMiDs is an anticancer drug that could bind to the other adapter protein CRBN of CUL4 [47, 48]. It connected the endogenous E3 ubiquitin of CUL4/CRBN. Based on the inhibition of enzyme activity, other proteins were generalized into new substrates $[48,50]$. However, studies found that the specific protein degradation of sulfonamide and lenalidomide was carried out independently, which led to clinically antimarrow tumor activity [29]. SPLAMs represent a new type of molecular gel-degrading agent after IMiDs. Specifically, they have a relatively weak receptor affinity [51]. After synergistically binding with DCAF15 and RBM39, they lead to the formation of a three-dimensional structure of DCAF15-DDB1-DDA1-RBM39 (RRM2), in which sulfonamides bind to the central helix of RBM39 (RRM2). It binds to DCAF15 (Fig. 3C, E), while DDA1 stabilizes the CRL4DCAF15 complex and promotes the recruitment of RBM39. In particular, RBM39 and indisulam had a substitution reaction during the interaction (Fig. 3B). We put the structures of the abovementioned four related but structurally different sulfonamides in the same substrate receptor of DCAF15 (Fig. 3D) to further illustrate the structural similarity of sulfonamides. In addition, the four sulfonamides clinically tested showed the same mechanism of action [52]. The degradation of RBM39 was essential for anticancer activity, and the anticancer activity of SPLAMs was in direct proportion to the expression of DCAF15 and the dependence of RBM39 $[52,53]$. Interestingly, the transcription of SPLAMs could downregulate RBM39, indicating that the expression of RBM39 might be negatively regulated by this protein [29]. In recent years, SPLAMs have been tested in phase II or III clinical trials in patients with metastatic cancer (Table 2), and have been found to progress relatively slowly in clinical trials due to their limited efficacy, with less than $15 \%$ of patients having a clinical response and an objective response rate of less than $40 \%$ in solid tumors [52]. The current clinical trials of SPLAMs mainly focus on leukemias and lymphomas that express DCAF15 protein at high levels, and have shown a certain degree of efficacy [54]. Therefore, the SPLAMs have potential clinical value in the treatment of cancer in the future, and they will also provide an important alternative splicing pathway opportunity for the growth of targeted cancer cells.

Indisulam (E7070) is a novel aryl sulfonamide with anticancer activity in vivo and in vitro discovered by Eisai pharmaceutical company in phenotype screening [55], and its antitumor activity in vivo is closely related to its effect on RBM39 [56]. With the increasing maturity of compound research, indisulam has shown effectiveness in a variety of clinical tumor models [57]. Moreover, it should be made clear that the anticancer agent indisulam is a target compound for the G1 phase of the cell cycle. As a carbonic anhydrase inhibitor, it inhibited the activation of CDK2 and cyclin $E$, causing the $G 1 / S$ conversion to be blocked, and then the cells died [56]. Studies revealed that indisulam connected RBM39 protein to ubiquitin mechanism through the adapter protein DCAF15, thereby inducing the degradation of RBM39 and further inhibiting cell proliferation [53]. Therefore, the current tumortargeted RNA splicing of indisulam is to recruit DCAF15 to induce the degradation of mRNA splicing factor RBM39 to target splicing [52]. At present, indisulam has conducted phase II clinical trials of patients with refractory or recurrent medullary malignancies, including indisulam combined with standard chemotherapy regimens, and has verified its clinical safety and disease stability $[54,58,59]$. More importantly, four phase I clinical trials have shown that the compound has nonlinear pharmacokinetics $[60,61]$. At the same time, the compound is also undergoing phase II clinical trials in Europe and the United States, but the biological basis of indisulam sensitivity is currently unclear, so it has only shown limited efficacy in some patients $[62,63]$.

\section{Proteasome inhibitor}

Targeted protein degradation is an emerging field in the development of small-molecule drugs. Cancer cells are highly sensitive to the cytotoxic effects of proteasome inhibition, making proteasome an important clinical target for targeted therapy of hematopoietic and lymphoid malignancies. As a kind of RNAbinding protein, RBM39 ubiquitination and proteasome degradation are essential for anticancer activity. Owing to its key role in the occurrence and maintenance of hematological malignancies, the degradation of proteasome has gradually become an important way for people to treat cancer. Nothing is more important than the fact that the reduction of RBM39 levels depends on the activity of the proteasome [52, 54]. Once it is degraded, it will lead to the abnormal splicing of pre-mRNA, which mainly includes intron retention and exon skipping, and leads to loss of cell viability and cell death [52]. At present, in the treatment of various hematological malignancies, a variety of small-molecule inhibitors have been developed and have successively entered the clinical trial stage (Table 3). Significantly, bortezomib, as the first proteasome inhibitor for the treatment of multiple myeloma, could block the indisulam-dependent degradation of RBM39 and showed better efficacy than other similar myeloma drugs [64]. It is considered that the approval of the drug set a precedent for the treatment of other malignant tumors [65]. Hereafter, bortezomib was used in combination with other drugs and found to have shown exciting results in the treatment of multiple myeloma and AML (Table 3). In addition, MLN4924 is a small-molecule inhibitor of UBA3, and it blocks the reduction of RBM39 induced by E7820 and the metabolism of Cullin RING ligases, so it is toxic to cells and is currently undergoing clinical trials [66]. It is reported that carfilzomib, a $\beta 5$ inhibitor, was approved by the FDA for the treatment of multiple myeloma in 2012, and is currently undergoing phase I clinical trials as a potential PI for the treatment of leukemia [67]. Subsequently, the combination therapy of bortezomib and IMiDs provided a good prognosis for MM, and other proteasome inhibitors are undergoing clinical trials [68]. In general, target protein degradation is an emerging field in drug development and application. Anticancer sulfonamides and proteasome inhibitors can promote cancer progression by inducing changes in pre-mRNA splicing.

\section{CONCLUDING REMARKS}

With the deepening of research, a growing number of scholars have generated a thought that RBM39, as a proto-oncogene, is not only closely related to the occurrence and development of a variety of malignant tumors, but also plays a crucial role in the clinical treatment of targeted drugs. As mentioned above, RBM39 
<smiles>NS(=O)(=O)c1ccc(S(=O)(=O)Nc2cccc3c(Cl)c[nH]c23)cc1</smiles>

Indisulam<smiles></smiles>

CQS<smiles>O=C(NS(=O)(=O)c1ccc(Br)o1)c1ccc(Cl)cc1Cl</smiles>

Tasisulam<smiles>Cc1ccc(NS(=O)(=O)c2cccc(C#N)c2)c2[nH]cc(C#N)c12</smiles>

E7820
B<smiles>Cc1ccc(NS(=O)(=O)c2ccc(CNC(=O)COCCOCCOCCNC(=S)Nc3ccc4c(c3)C3(OC4=O)c4ccc(O)cc4Oc4cc(O)ccc43)cc2)c2[nH]cc(C#N)c12</smiles>

C

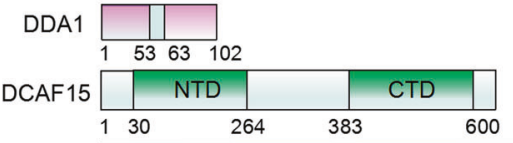

D

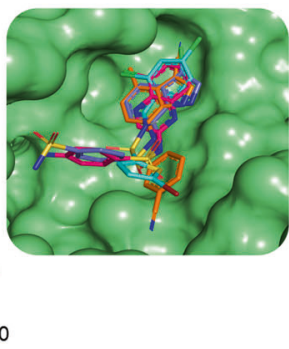

E

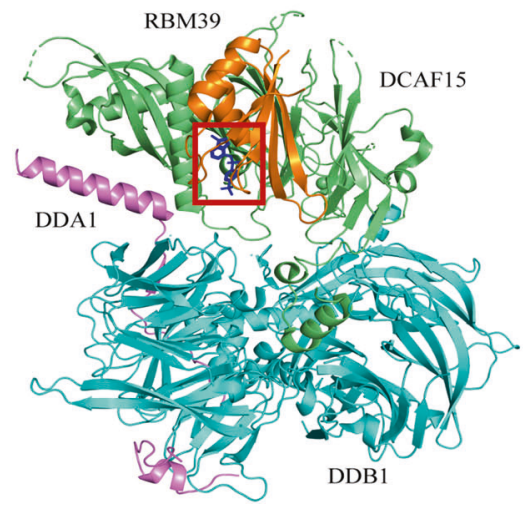

Fig. 3 Structure of the complex of DDA1-DDB1-DCAF15-RBM39 with SPLAMs. A Chemical structures of indisulam, CQS, tasisulam and E7820. B Substitution reaction in the RBM39-indisulam interaction. C Domain representation of the proteins present in the complex. D The surface of DCAF15 is represented as green, and CQS, E7820, indisulam and tasisulam are represented as rods in red, orange, blue and cyan respectively. E Overall quaternary structure of human DCAF15-DDB1-DDA1-RBM39 (RRM2) in complex with aryl sulfonamides. DCAF15 is shown in green, DDB1 in blue, DDA1 in purple and RBM39 (RRM2) in orange. The aryl sulfonamide-binding site between DCAF15 and RBM39 is outlined in red.

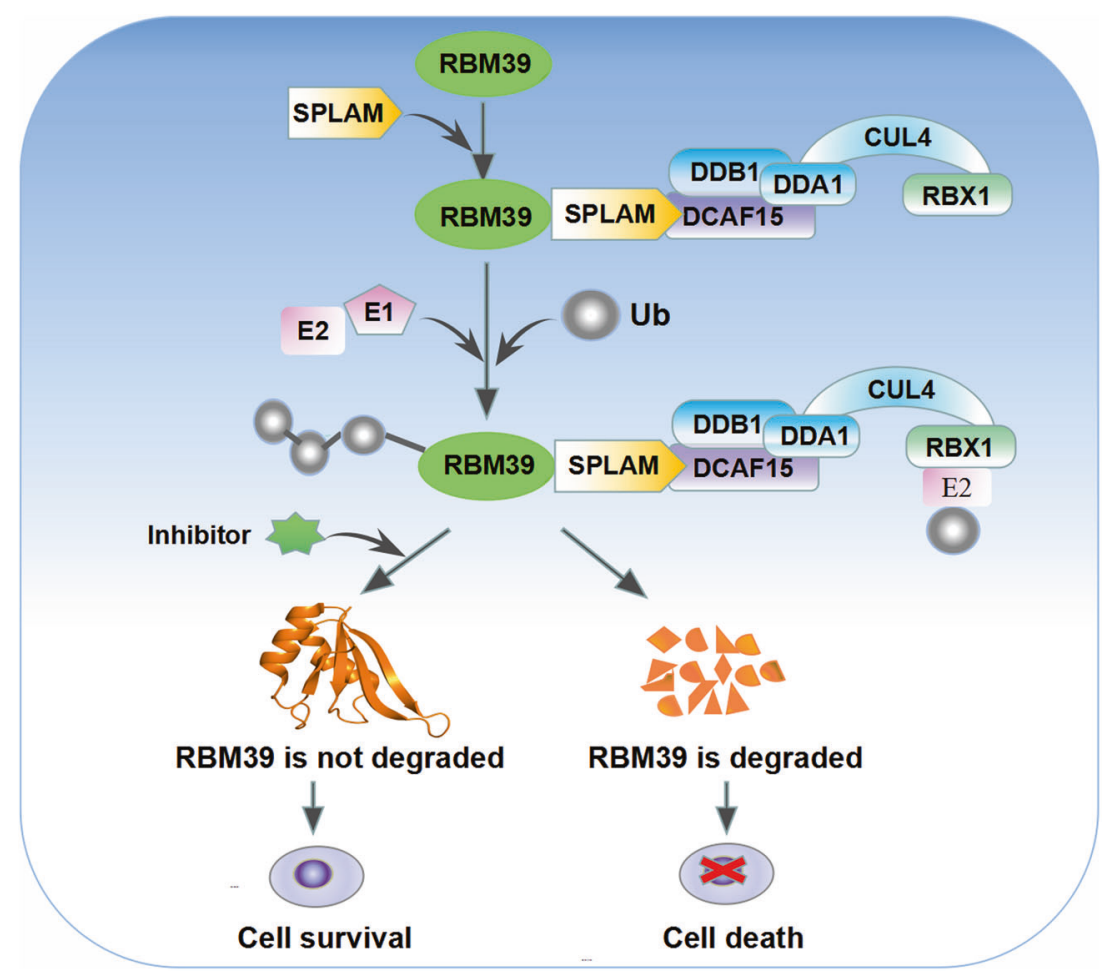

Fig. 4 Schematic diagram of SPLAMs promoting degradation of RBM39. SPLAMs promote the interaction between RBM39 and DCAF15 E3 ligase substrate receptor, leading to ubiquitination of RBM39 and proteasome-mediated degradation, while proteasome inhibitors can prevent RBM39 degradation.

indirectly participates in the growth and progression of tumors by regulating the transcription of many tumor-related genes, protein translation, and selective splicing, which provides new ideas and guiding significance for clarifying the pathogenesis of RBM39 in cancer. Since RBM39 was identified as the target of SPLAMs, the degradation of targeted proteins has gradually become an emerging field in the development of small-molecule drugs. It not only provides novel biomarkers for disease progression, but 
Table 2. Clinical trials of aryl sulfonamides.

\begin{tabular}{|c|c|c|c|c|}
\hline Type & NCT Number & Condition & Phase & Start Date \\
\hline \multirow[t]{7}{*}{ Indisulam } & NCT00165594 & Gastric cancer & Phase 1, Phase 2 & February 2005 \\
\hline & NCT00165867 & Colorectal cancer & Phase 2 & April 2005 \\
\hline & NCT01692197 & Leukemia & Phase 2 & February 2013 \\
\hline & NCT00059735 & Kidney Neoplasms, Carcinoma, Adenocarcinoma, & Phase 2 & May 2002 \\
\hline & NCT00165880 & Breast Cancer & Phase 2 & December 2004 \\
\hline & NCT00165854 & Colorectal cancer & Phase 2 & March 2003 \\
\hline & NCT00080197 & Breast neoplasms & Phase 2 & February 2004 \\
\hline \multirow[t]{4}{*}{ E7820 } & NCT01133990 & Colorectal cancer & Phase 1, Phase 2 & April 2010 \\
\hline & NCT00309179 & Advanced colorectal cancer & Phase 2 & September 2007 \\
\hline & NCT00078637 & Malignant neoplasms, lymphoma & Phase 1 & January 2004 \\
\hline & NCT01347645 & Colon cancer, Rectal cancer & Phase 1, Phase 2 & September 2011 \\
\hline & NCT01258348 & Metastatic renal cell cancer & Phase 1 & July 2008 \\
\hline & NCT01214668 & Solid tumors & Phase 1 & January 2009 \\
\hline & NCT00383292 & Metastatic melanoma & Phase 2 & November 2006 \\
\hline & NCT00992225 & Breast cancer & Phase 2 & September 2009 \\
\hline & NCT00363766 & Non-small-cell lung Cancer & Phase 2 & September 2006 \\
\hline & NCT01284335 & Advanced solid tumors & Phase 1 & July 2008 \\
\hline & NCT01006252 & Melanoma & Phase 3 & December 2009 \\
\hline & NCT00718159 & Acute myeloid leukemia & Phase 1 & August 2008 \\
\hline & NCT01185548 & Lymphoma, Advanced cancer & Phase 1 & July 2010 \\
\hline
\end{tabular}

Table 3. Proteasome small molecule inhibitor of B5 protease for cancer treatment.

\begin{tabular}{|c|c|}
\hline Modulators & Condition \\
\hline $\begin{array}{l}\text { Bortezomib + Daunorubicin + } \\
\text { Cytarabine }\end{array}$ & Acute myeloid leukemia \\
\hline Bortezomib + Decytabine & Acute myeloid leukemia, Myeloma \\
\hline \multicolumn{2}{|l|}{ Bortezomib + SAHA } \\
\hline Bortezomib & Myeloma malignancy \\
\hline arlfizomib & Myeloma malignancy \\
\hline xazomib & Myeloma malignancy \\
\hline Marizomib & $\begin{array}{l}\text { Myeloma malignancy, mantle cell ly } \\
\text { lymphocytic leukemia, colorectal an }\end{array}$ \\
\hline Delanzomib & Myeloma malignancy and other $n$ \\
\hline \multicolumn{2}{|c|}{$\begin{array}{l}\text { also affects the splicing of pre-mRNA subsets through the } \\
\text { degradation of drugs. Therefore, CRISPR-Cas9-based DCAF15 } \\
\text { gene knockout and single amino acid substitution of RBM39 can } \\
\text { resist the degradation of RBM } 39 \text { induced by sulfonamides, } \\
\text { suggesting that RBM39 degradation is a key biochemical indicator } \\
\text { of the anticancer properties of these compounds. At present, there } \\
\text { are still many challenging problems to be solved. First, although } \\
\text { the critical role of RBM39 in the occurrence and development of } \\
\text { cancer has been supported by many reports, the current } \\
\text { understanding of the mechanism of SPLAMs may be insufficient. } \\
\text { Furthermore, although the safety of SPLAMs has been confirmed }\end{array}$} \\
\hline
\end{tabular}

\begin{tabular}{ll} 
Clinical Stage & Refs \\
\hline Phase 1 & {$[1]$} \\
\hline Phase 2 & {$[2]$} \\
\hline Phase 2 suspended & {$[3]$} \\
\hline Phase 1 & {$[4]$} \\
\hline Phase 1 & {$[5]$} \\
\hline Phase 1 & {$[6]$} \\
\hline Phase 3, recruiting & {$[7]$} \\
\hline $\begin{array}{l}\text { Phase 1, Phase 2, } \\
\text { terminated }\end{array}$ & {$[8]$} \\
\hline
\end{tabular}

also affects the splicing of pre-mRNA subsets through the degradation of drugs. Therefore, CRISPR-Cas9-based DCAF15 gene knockout and single amino acid substitution of RBM39 can resist the degradation of RBM39 induced by sulfonamides, suggesting that RBM39 degradation is a key biochemical indicator of the anticancer properties of these compounds. At present, there are still many challenging problems to be solved. First, although the critical role of RBM39 in the occurrence and development of understanding of the mechanism of SPLAMs may be insufficient. Furthermore, although the safety of SPLAMs has been confirmed in many previous phase I and phase II clinical trials, it is still unclear whether RBM39 is an effective target of SPLAMs. Hence, it is necessary for future clinical studies to in-depth study the preferential effect of RBM39 degradation in cancer cells based on the spliceosome genotype, and to further determine the exact function of RBM39 in splicing. Although SPLAMs have only shown moderate efficacy in clinical trials so far, the biological basis of sensitivity to these drugs is still unclear, so they have only shown limited efficacy in some patients. Will SPLAMs be used in the treatment and research of more clinical patients in the future to improve the potential clinical efficacy of SPLAMs? Can SPLAM 
derivatives recruit new substrates other than RBM39 to join CUL4-DCAF15? Furthermore, so many questions are very necessary in the future research field to determine and further clarify the structural basis of CRL4-DCAF15 to identify sulfonamide degradation. The in-depth study of these sulfonamides may expand our understanding of the biological functions of RBM39, thereby guiding us to choose the correct target type that is more sensitive to these drugs for better application in cancer treatment. Taken together, further clinical trials will help determine the use of drugs targeting RBM39 activity in cancer therapy.

\section{REFERENCES}

1. Imai H, Chan EK, Kiyosawa K, Fu XD, Tan EM. Novel nuclear autoantigen with splicing factor motifs identified with antibody from hepatocellular carcinoma. J Clin Investig. 1993;92:2419-26.

2. Dowhan DH, Hong EP, Auboeuf D, Dennis AP, Wilson MM, Berget SM, et al. Steroid hormone receptor coactivation and alternative RNA splicing by U2AF65related proteins CAPERalpha and CAPERbeta. Mol Cell. 2005;17:429-39.

3. Chai Y, Liu X, Dai L, Li Y, Liu M, Zhang JY. Overexpression of HCC1/CAPERa may play a role in lung cancer carcinogenesis. Tumour Biol J Int Soc Oncodev Biol Med. 2014;35:6311-7.

4. Campbell MC, Pontiggia L, Russell AY, Schwarting R, Camacho J, Jasmin JF, et al. CAPER as a therapeutic target for triple negative breast cancer. Oncotarget 2018;9:30340-54.

5. Sillars-Hardebol AH, Carvalho B, Beliën JA, de Wit M, Delis-van Diemen PM, Tijssen $M$, et al. CSE1L, DIDO1 and RBM39 in colorectal adenoma to carcinoma progression. Cell Oncol. 2012;35:293-300.

6. Mercier I, Gonzales DM, Quann K, Pestell TG, Molchansky A, Sotgia F, et al. CAPER, a novel regulator of human breast cancer progression. Cell Cycle. 2014;13:1256-64.

7. Lalaoui N, Merino D, Giner G, Vaillant F, Chau D, Liu L, et al. Targeting triplenegative breast cancers with the Smac-mimetic birinapant. Cell Death Differ. 2020;27:2768-80.

8. Dutta J, Fan G, Gélinas C. CAPERalpha is a novel Rel-TAD-interacting factor that inhibits lymphocyte transformation by the potent Rel/NF-kappaB oncoprotein v-Rel. J Virol. 2008;82:10792-802.

9. Huang G, Zhou Z, Wang H, Kleinerman ES. CAPER-a alternative splicing regulates the expression of vascular endothelial growth factor ${ }_{165}$ in Ewing sarcoma cells. Cancer 2012;118:2106-16.

10. Královicová J, Ševcíková I, Stejskalová E, Obuca M, Hiller M, Stanek D, et al. PUF60activated exons uncover altered $3^{\prime}$ splice-site selection by germline missense mutations in a single RRM. Nucleic Acids Res. 2018;46:6166-87.

11. Loerch S, Maucuer A, Manceau V, Green MR, Kielkopf CL. Cancer-relevant splicing factor CAPERa engages the essential splicing factor SF3b155 in a specific ternary complex. J Biol Chem. 2014;289:17325-37.

12. Lebon G, Langmead CJ, Tehan BG, Hulme EC. Mutagenic mapping suggests a novel binding mode for selective agonists of $M 1$ muscarinic acetylcholine receptors. Mol Pharmacol. 2009;75:331-41.

13. Cazalla D, Newton K, Cáceres JF. A novel SR-related protein is required for the second step of pre-mRNA splicing. Mol Cell Biol. 2005;25:2969-80.

14. Jung DJ, Na SY, Na DS, Lee JW. Molecular cloning and characterization of CAPER, a novel coactivator of activating protein-1 and estrogen receptors. J Biol Chem. 2002;277:1229-34.

15. Rappsilber J, Ryder U, Lamond Al, Mann M. Large-scale proteomic analysis of the human spliceosome. Genome Res. 2002;12:1231-45.

16. Su Al, Wiltshire T, Batalov S, Lapp H, Ching KA, Block D, et al. A gene atlas of the mouse and human protein-encoding transcriptomes. Proc Natl Acad Sci USA. 2004;101:6062-7.

17. Schulien I, Hockenjos B, Schmitt-Graeff A, Perdekamp MG, Follo M, Thimme R, et al. The transcription factor C-Jun/AP-1 promotes liver fibrosis during nonalcoholic steatohepatitis by regulating Osteopontin expression. Cell Death Differ. 2019;26:1688-99.

18. Ramakrishna M, Williams LH, Boyle SE, Bearfoot JL, Sridhar A, Speed TP, et al. Identification of candidate growth promoting genes in ovarian cancer through integrated copy number and expression analysis. PloS ONE. 2010;5:e9983.

19. Mai S, Qu X, Li P, Ma Q, Cao C, Liu X. Global regulation of alternative RNA splicing by the SR-rich protein RBM39. Biochim Biophys Acta. 2016;1859:1014-24.

20. Mai S, Qu X, Li P, Ma Q, Liu X, Cao C. Functional interaction between nonreceptor tyrosine kinase c-Abl and SR-Rich protein RBM39. Biochem Biophys Res Commun. 2016;473:355-60.

21. Kang YK, Putluri N, Maity S, Tsimelzon A, Ilkayeva O, Mo Q, et al. CAPER is vital for energy and redox homeostasis by integrating glucose-induced mitochondrial functions via ERR-a-Gabpa and stress-induced adaptive responses via NF-KBcMYC. PLoS Genet. 2015;11:e1005116.
22. Kumar PP, Emechebe U, Smith R, Franklin S, Moore B, Yandell M, et al. Coordinated control of senescence by IncRNA and a novel T-box3 co-repressor complex. eLife. 2014;3:e02805.

23. Moore MJ, Wang Q, Kennedy CJ, Silver PA. An alternative splicing network links cell-cycle control to apoptosis. Cell 2010;142:625-36.

24. Di C, Syafrizayanti, Zhang Q, Chen Y, Wang Y, Zhang X, et al. Function, clinical application, and strategies of pre-mRNA splicing in cancer. Cell Death Differ. 2019;26:1181-94.

25. Stepanyuk GA, Serrano P, Peralta E, Farr CL, Axelrod HL, Geralt M, et al. UHM-ULM interactions in the RBM39-U2AF65 splicing-factor complex. Acta Cryst. 2016;72:497-511.

26. Shepard PJ, Hertel KJ. The SR protein family. Genome Biol. 2009;10:242.

27. Ellis JD, Llères $D$, Denegri $M$, Lamond Al, Cáceres JF. Spatial mapping of splicing factor complexes involved in exon and intron definition. J Cell Biol. 2008;181:921-34.

28. Prigge JR, Iverson SV, Siders AM, Schmidt EE. Interactome for auxiliary splicing factor U2AF(65) suggests diverse roles. Biochim Biophys Acta. 2009; 1789:487-92.

29. Uehara T, Minoshima Y, Sagane K, Sugi NH, Mitsuhashi KO, Yamamoto N, et al. Selective degradation of splicing factor CAPERa by anticancer sulfonamides. Nat Chem Biol. 2017;13:675-80.

30. Sharma S, Falick AM, Black DL. Polypyrimidine tract binding protein blocks the $5^{\prime}$ splice site-dependent assembly of U2AF and the prespliceosomal E complex. Mol Cell. 2005;19:485-96.

31. Nordin A, Larsson E, Holmberg M. The defective splicing caused by the ISCU intron mutation in patients with myopathy with lactic acidosis is repressed by PTBP1 but can be derepressed by IGF2BP1. Hum Mutat. 2012;33:467-70.

32. Faherty $N$, Benson $M$, Sharma $E$, Lee $A$, Howarth $A$, Lockstone $H$, et al. Negative autoregulation of BMP dependent transcription by SIN3B splicing reveals a role for RBM39. Sci Rep. 2016;6:28210.

33. Martínez-Arribas F, Agudo D, Pollán M, Gómez-Esquer F, Díaz-Gil G, Lucas R, et al. Positive correlation between the expression of X-chromosome RBM genes (RBMX, RBM3, RBM10) and the proapoptotic Bax gene in human breast cancer. J Cell Biochem. 2006;97:1275-82.

34. Chilewski SD, Bhosale D, Dees S, Hutchinson I, Trimble R, Pontiggia L, et al. Development of CAPER peptides for the treatment of triple negative breast cancer. Cell Cycle. 2020;19:432-47.

35. Mercier I, Casimiro MC, Zhou J, Wang C, Plymire C, Bryant KG, et al. Genetic ablation of caveolin-1 drives estrogen-hypersensitivity and the development of DCIS-like mammary lesions. Am J Pathol. 2009;174:1172-90.

36. Döhner H, Estey E, Grimwade D, Amadori S, Appelbaum FR, Büchner T, et al. Diagnosis and management of AML in adults: 2017 ELN recommendations from an international expert panel. Blood 2017;129:424-47.

37. Katsumura KR, Ong IM, DeVilbiss AW, Sanalkumar R, Bresnick EH. GATA factordependent positive-feedback circuit in acute myeloid leukemia cells. Cell Rep. 2016;16:2428-41.

38. Seiler M, Yoshimi A, Darman R, Chan B, Keaney G, Thomas M, et al. H3B-8800, an orally available small-molecule splicing modulator, induces lethality in spliceosome-mutant cancers. Nat Med. 2018;24:497-504.

39. Thomas R, Majeti R. No matter how you splice it, RBM39 inhibition targets spliceosome mutant AML. Cancer Cell. 2019;35:337-9.

40. Saha S, Murmu KC, Biswas M, Chakraborty S, Basu J, Madhulika S, et al. Transcriptomic analysis identifies RNA binding proteins as putative regulators of myelopoiesis and leukemia. Front Oncol. 2019;9:692.

41. Wang E, Lu SX, Pastore A, Chen X, Imig J, Chun-Wei Lee S, et al. Targeting an RNA-binding protein network in acute myeloid leukemia. Cancer Cell. 2019;35:369-84.e7.

42. Bangur CS, Switzer A, Fan L, Marton MJ, Meyer MR, Wang T. Identification of genes over-expressed in small cell lung carcinoma using suppression subtractive hybridization and cDNA microarray expression analysis. Oncogene 2002;21:3814-25.

43. Maiso P, Huynh D, Moschetta M, Sacco A, Aljawai Y, Mishima Y, et al. Metabolic signature identifies novel targets for drug resistance in multiple myeloma. Cancer Res. 2015;75:2071-82.

44. Tong J, Xu X, Zhang Z, Ma C, Xiang R, Liu J, et al. Hypoxia-induced long noncoding RNA DARS-AS1 regulates RBM39 stability to promote myeloma malignancy. Haematologica 2020;105:1630-40.

45. Dai L, Peng XX, Tan EM, Zhang JY. Tumor-associated antigen CAPERa and microvessel density in hepatocellular carcinoma. Oncotarget 2016;7:16985-95.

46. Sillars-Hardebol AH, Carvalho B, Tijssen M, Beliën JA, de Wit M, Delis-van Diemen PM, et al. TPX2 and AURKA promote 20q amplicon-driven colorectal adenoma to carcinoma progression. Gut. 2012;61:1568-75.

47. Lu G, Middleton RE, Sun H, Naniong M, Ott CJ, Mitsiades CS, et al. The myeloma drug lenalidomide promotes the cereblon-dependent destruction of Ikaros proteins. Science. 2014;343:305-9. 
48. Krönke J, Fink EC, Hollenbach PW, MacBeth KJ, Hurst SN, Udeshi ND, et al. Lenalidomide induces ubiquitination and degradation of CK1a in del(5q) MDS. Nature 2015;523:183-8.

49. Du X, Volkov OA, Czerwinski RM, Tan H, Huerta C, Morton ER, et al. Structural basis and kinetic pathway of RBM39 recruitment to DCAF15 by a sulfonamide molecular glue E7820. Structure. 2019;27:1625-33.e3.

50. Krönke J, Udeshi ND, Narla A, Grauman P, Hurst SN, McConkey M, et al. Lenalidomide causes selective degradation of IKZF1 and IKZF3 in multiple myeloma cells. Science. 2014;343:301-5.

51. Winter GE, Buckley DL, Paulk J, Roberts JM, Souza A, Dhe-Paganon S, et al. DRUG DEVELOPMENT. Phthalimide conjugation as a strategy for in vivo target protein degradation. Science. 2015;348:1376-81.

52. Han T, Goralski M, Gaskill N, Capota E, Kim J, Ting TC, et al. Anticancer sulfonamides target splicing by inducing RBM39 degradation via recruitment to DCAF15. Science. 2017;356:eaal3755.

53. Bussiere DE, Xie L, Srinivas $H$, Shu W, Burke A, Be C, et al. Structural basis of indisulam-mediated RBM39 recruitment to DCAF15 E3 ligase complex. Nat Chem Biol. 2020;16:15-23.

54. Ting TC, Goralski M, Klein K, Wang B, Kim J, Xie Y, et al. Aryl sulfonamides degrade RBM39 and RBM23 by recruitment to CRL4-DCAF15. Cell Rep. 2019;29:1499-510.e6

55. Owa T, Yoshino H, Okauchi T, Yoshimatsu K, Ozawa Y, Sugi NH, et al. Discovery of novel antitumor sulfonamides targeting G1 phase of the cell cycle. J Med Chem. 1999;42:3789-99.

56. Fukuoka K, Usuda J, Iwamoto Y, Fukumoto H, Nakamura T, Yoneda T, et al. Mechanisms of action of the novel sulfonamide anticancer agent E7070 on cell cycle progression in human non-small cell lung cancer cells. Invest New Drugs. 2001;19:219-27.

57. Ozawa Y, Sugi NH, Nagasu T, Owa T, Watanabe T, Koyanagi N, et al. E7070, a novel sulphonamide agent with potent antitumour activity in vitro and in vivo. Eur J Cancer. 2001;37:2275-82.

58. Jia X, Pan L, Zhu M, Hu H, Zhai L, Liu J, et al. pSILAC method coupled with two complementary digestion approaches reveals PRPF39 as a new E7070-dependent DCAF15 substrate. J Proteom. 2020;210:103545.

59. Assi R, Kantarjian HM, Kadia TM, Pemmaraju N, Jabbour E, Jain N, et al. Final results of a phase 2 , open-label study of indisulam, idarubicin, and cytarabine in patients with relapsed or refractory acute myeloid leukemia and high-risk myelodysplastic syndrome. Cancer 2018;124:2758-65.

60. Punt CJ, Fumoleau P, van de Walle B, Faber MN, Ravic M, Campone M, et al. Phase I and pharmacokinetic study of E7070, a novel sulfonamide, given at a daily times five schedule in patients with solid tumors. A study by the EORTC-early clinical studies group (ECSG). Ann. Oncol.2001;12:1289-93.

61. Dittrich $C$, Dumez H, Calvert $H$, Hanauske A, Faber M, Wanders J, et al. Phase I and pharmacokinetic study of E7070, a chloroindolyl-sulfonamide anticancer agent, administered on a weekly schedule to patients with solid tumors. Clin Cancer Res. 2003;9:5195-204.

62. Talbot DC, von Pawel J, Cattell E, Yule SM, Johnston C, Zandvliet AS, et al. A randomized phase II pharmacokinetic and pharmacodynamic study of indisulam as second-line therapy in patients with advanced non-small cell lung cancer. Clin Cancer Res. 2007;13:1816-22.

63. Yamada Y, Yamamoto N, Shimoyama T, Horiike A, Fujisaka Y, Takayama K, et al. Phase I pharmacokinetic and pharmacogenomic study of E7070 administered once every 21 days. Cancer Sci. 2005;96:721-8.

64. Hungria VTM, Crusoé EQ, Bittencourt RI, Maiolino A, Magalhães RJP, Sobrinho JDN, et al. New proteasome inhibitors in the treatment of multiple myeloma. Hematol Transfus Cell Ther. 2019:41:76-83.

65. Chen D, Frezza M, Schmitt S, Kanwar J, Dou QP. Bortezomib as the first proteasome inhibitor anticancer drug: current status and future perspectives. Curr Cancer Drug Targets. 2011;11:239-53.
66. Soucy TA, Smith PG, Milhollen MA, Berger AJ, Gavin JM, Adhikari S, et al. An inhibitor of NEDD8-activating enzyme as a new approach to treat cancer. Nature 2009;458:732-6.

67. Swift L, Jayanthan A, Ruan Y, Anderson R, Boklan J, Trippett T, et al. Targeting the proteasome in refractory pediatric leukemia cells: characterization of effective cytotoxicity of carfilzomib. Target Oncol. 2018;13:779-93.

68. Richardson PG, Xie W, Jagannath S, Jakubowiak A, Lonial S, Raje NS, et al. A phase 2 trial of lenalidomide, bortezomib, and dexamethasone in patients with relapsed and relapsed/refractory myeloma. Blood 2014;123:1461-9.

\section{ACKNOWLEDGEMENTS}

This work was supported by grants from the National Key R\&D project of the Chinese Ministry of Science and Technology [2018YFE0205100], the Key Program of the National Natural Science Foundation of China [U1632270], and the National Natural Science Foundation of China [11675234].

\section{AUTHOR CONTRIBUTIONS}

CX Di, $\mathrm{H}$ Zhang, and $\mathrm{Q} \mathrm{Li}$, as co-corresponding authors, designed the framework for this review; DP Zhao and ZH Dou were responsible for the retrieval and collation of relevant literature; XD Xie, $\mathrm{HY} \mathrm{Li}$, and HY Yang mainly provided suggestions and new ideas in the study of RBM39; CP Xu mainly wrote the paper; XH Chen and XT Zhang modified the paper; all authors approved the final version.

\section{COMPETING INTERESTS}

The authors declared no competing interest.

\section{ADDITIONAL INFORMATION}

Supplementary information The online version contains supplementary material available at https://doi.org/10.1038/s41420-021-00598-7.

Correspondence and requests for materials should be addressed to Q.L., H.Z. or C.D.

Reprints and permission information is available at http://www.nature.com/reprints

Publisher's note Springer Nature remains neutral with regard to jurisdictional claims in published maps and institutional affiliations.

\begin{abstract}
Access This article is licensed under a Creative Commons Attribution 4.0 International License, which permits use, sharing, adaptation, distribution and reproduction in any medium or format, as long as you give appropriate credit to the original author(s) and the source, provide a link to the Creative Commons license, and indicate if changes were made. The images or other third party material in this article are included in the article's Creative Commons license, unless indicated otherwise in a credit line to the material. If material is not included in the article's Creative Commons license and your intended use is not permitted by statutory regulation or exceeds the permitted use, you will need to obtain permission directly from the copyright holder. To view a copy of this license, visit http://creativecommons. org/licenses/by/4.0/.
\end{abstract}

(c) The Author(s) 2021 